\title{
Polymer Effect on Molecular Recognition. Enhancement of Molecular-Shape Selectivity for Polycyclic Aromatic Hydrocarbons by Poly(acrylonitrile)
}

\author{
Makoto TAKafuji, Wei Dong, Yoshihiro Goto, Toshihiko SAKURAI, \\ Shoji NAGAOKA, ${ }^{*}$ and Hirotaka IHARA ${ }^{\dagger}$ \\ Department of Applied Chemistry \& Biochemistry, Faculty of Engineering, \\ Kumamoto University, Kumamoto 860-8555, Japan \\ *Kumamoto Industrial Research Institute, Kumamoto 862-0901, Japan
}

(Received December 10, 2001; Accepted April 25, 2002)

\begin{abstract}
Poly(acrylonitrile) immobilized onto porous silica $\left(\mathrm{Sil}_{-} \mathrm{AN}_{n}\right)$ was prepared to evaluate the effect of polymerization degree of poly(acrylonitrile) on selective interaction with polycyclic aromatic hydrocarbons. The HPLC using the packed column $\left(\mathrm{Sil}_{-} \mathrm{AN}_{n}\right)$ and an aqueous solution as a mobile phase showed higher selectivity for structural isomers of polycyclic aromatic hydrocarbons compared with simply cyanopropylated silica (Sil-CN) and alkylated silica $\left(\mathrm{Sil}_{-} \mathrm{C}_{4}\right)$. It was considered that silica-supported poly(acrylonitrile) recognized molecular aromaticity of $\pi$-electron containing compounds rather than molecular hydrophobicity. Furthermore, similar results were obtained in the selectivity towards geometrical isomers such as trans- and cis-stilbenes or triphenylene and $o$-terphenyl and structural isomers such as $o-, m-, p$-terphenyls. Also the separation factors increased with an increase in polymerization degree of $\mathrm{AN}_{n}$. This paper discusses that polymeric structures enhance the selectivity.

KEY WORDS Polymer Grafting Silica / High Performance Liquid Chromatography (HPLC) /

Multiple $\pi-\pi$ Interactions / CN- $\pi-$ to-benzene $-\pi$ Interaction / Molecular Aromaticity / Geometrical Isomers / Structural Isomers /
\end{abstract}

Specific behaviors of many polymers are based on their configurations and conformations. The chemical structures of monomer units and their arrangements influence on the conformational structures of polymer chain and then closely relate to their functions whose are carried out by multiple interactions through accumulation of their functional groups. In biological systems, there are many examples of protein polymers, the structures of which determine the biological functions. The molecular assemblies such as bio-membranes also show specific behaviors in bio-systems.

On the other hand, synthetic polymers are used as many industrial materials such as films, fibers and adhesions. It is well-known that crystallinity and polymerization degree influence the properties of polymers. Many researchers have reported physical and chemical properties of polymers with functional groups in their main and side chains because it is important to mimic the functions of biopolymers using simply synthetic polymers. This will expand possible applications of polymers.

We have investigated the hybridization between silica gels and polymers ${ }^{1-10}$ such as polystyrene, ${ }^{1}$ poly(acrylonitrile), ${ }^{6}$ and poly(methyl acrylate) ${ }^{3}$ which were applied to separation. These polymers possess $\pi$ electrons to be used as a $\pi-\pi$ interaction source and thus have been applied as packing materials for liquid chromatography with $\pi$-electron recognition. As a re-

${ }^{\dagger}$ To whom correspondence should be addressed. sult, we have found that the polymer-immobilized silica showed much better selectivity for structural isomers of polycyclic aromatic hydrocarbons than the corresponding monomer-immobilized silica. In this paper, we wish to report the effects of polymerization degree of polymers on separation behavior of polycyclic aromatic hydrocarbons by using poly(acrylonitrile).

\section{EXPERIMENTAL}

\section{Materials}

Acrylonitrile (AN; Nacalai tesque, Japan) was distilled and the fraction boiling at $72-74^{\circ} \mathrm{C}$ was used. 3Mercaptopropyltrimethoxysilane (MPS) as telogen was purchased from Chisso Co., Japan. Azobisisobutyronitrile (AIBN) as an initiator was purified by recrystallization from methanol. A typical telomerization procedure of poly(acrylonitrile), $\mathrm{AN}_{n}$, where $n$ is the average of polymerization degree, was as follows: AN $(35 \mathrm{~mL}, 532 \mathrm{mmol})$ and MPS $(10 \mathrm{~mL}, 53 \mathrm{mmol})$ were dissolved in ethanol $(200 \mathrm{~mL})$. AIBN $(0.14 \mathrm{~g}, 0.5 \mathrm{wt} \%)$ was added to the solution at $60^{\circ} \mathrm{C}$. The mixture was stirred for $72 \mathrm{~h}$ at $60^{\circ} \mathrm{C}$ under $\mathrm{N}_{2}$ gas atmosphere. After the reaction, the white precipitates obtained were gathered by concentration and filtration, washed successively with ethanol and ether, and then dried in vacuo. The polymerization degree $(n)$ of $\mathrm{AN}_{n}$ was determined by ${ }^{1} \mathrm{H}$ NMR spectroscopy (in $d_{6}$-dimethylsulfoxide, $\delta=$ $2.05 \mathrm{ppm}\left(\mathrm{C}_{2} \mathrm{CH}-\mathrm{CN}\right), \delta=3.15 \mathrm{ppm}\left(\mathrm{CH}_{2} \mathrm{C} \underline{\mathrm{H}}-\mathrm{CN}\right)$, 
and $\left.\delta=3.55 \mathrm{ppm}\left(\mathrm{SiOCH}_{3}\right)\right)$.

$\mathrm{AN}_{n}$ was readily introduced onto porous silica particle by mixing in dimethylformamide at $80^{\circ} \mathrm{C}$. YMC 120-S5 silica (diameter $5 \mu \mathrm{m}$, pore size $120 \AA, 295 \mathrm{~m}^{2}$ $\mathrm{g}^{-1}$ ) was used as porous silica. The resulting particles were washed successively with dimethylformamide and ether. The amount of $\mathrm{AN}_{n}$ introduced onto silica was determined by elemental analysis.

Wakopak (Wakosil 5CN, $4.6 \mathrm{~mm}$ i.d. $\times 250 \mathrm{~mm}$ ) was used as a CN-containing silica (Sil-CN). Mightysil (RP-4 GP, $4.6 \mathrm{~mm}$ i.d. $\times 250 \mathrm{~mm}$ ) was used as a simplyhydrophobized silica $\left(\mathrm{Sil}_{-} \mathrm{C}_{4}\right)$.

All aromatic hydrocarbons as a sample were commercially obtained and used without further purification. Benzene, anthracene, pyrene, triphenylene and terphenyl isomers were purchased from Nacalai tesque (Kyoto, Japan). Naphthacene was purchased from Tokyo Kasei Kogyo Co., Ltd. (Tokyo, Japan). Naphthalene, dinitrobenzene isomers and dinitrotoluene isomers were purchased from Kanto Kagaku (Tokyo, Japan). Chrysene and stilbene isomers were purchased from Sigma-Aldrich Co. (St. Louis, MO). Benz[a]anthracene was purchased from Kishida Chemical Co., Ltd. (Osaka, Japan).

\section{Measurements}

The polymer-grafted silica $\left(\mathrm{Sil}_{-} \mathrm{AN}_{n}\right)$ was packed into a stainless steel column $(4.6 \mathrm{~mm}$ i.d. $\times 250 \mathrm{~mm})$ using a hexanol-chloroform mixture. The liquid chromatographic properties were examined using methanolwater mixtures as mobile phases. The chromatograph included a JASCO PU-980 intelligent HPLC pump, and a JASCO MD-910 multiwavelength detector. JASCOBORWIN (Ver. 1.5) software was used for system control and data analysis. 10 micro-liters of the sample dissolved in methanol was injected through a HAMILTON 80365 injector. The mobile phases were methanolwater $(6: 4)$ for Sil-CN and Sil-AN ${ }_{n}$ columns, and methanol-water (7:3) for $\mathrm{Sil}_{-} \mathrm{C}_{4}$ column. Chromatography was carried out at flow-rate of $1.0 \mathrm{~mL} \mathrm{~min}^{-1}$. The retention factor $(k)$ was determined by $\left(t_{\mathrm{e}}-t_{0}\right) / t_{0}$, where $t_{\mathrm{e}}$ and $t_{0}$ are retention time of samples and methanol, respectively. The separation factor $(\alpha)$ was defined by the ratio of retention factor.

Water-1-octanol partition coefficient $(\log P)$ was determined by retention factor with octadecylated silica, ODS (Inertsil ODS, $4.6 \mathrm{~mm}$ i. d. $\times 300 \mathrm{~mm}$, GL Science Co., Ltd.) : $\log P=3.2622+4.208 \log k^{11}$

\section{RESULTS AND DISCUSSION}

\section{Preparation of Silica-Supported Poly(acrylonitrile)}

Poly(acrylonitrile) with a terminal trimethoxysilyl group $\left(\mathrm{AN}_{n}\right)$ was prepared by telomerization of acrylonitrile with 3-mercaptopropyltrimethoxysilane. The average of polymerization degree was easily controlled by adjustment of the molar ratio. As a result, $\mathrm{AN}_{n}$ with $n=3$ and 21 were prepared for our purpose.

The $\mathrm{AN}_{n}$ was immobilized onto porous silica. This was done through the reaction between the terminal trimethoxysilyl groups of $\mathrm{AN}_{n}$ and silanol groups from silica. The resulting silica successive washing with dimethylformamide showed no change in weight. IR spectroscopy showed the specific absorption due to $\mathrm{CN}$ groups $\left(v_{\mathrm{C}-\mathrm{N}}, 2273 \mathrm{~cm}^{-1}\right)$. The elemental analysis showed that $11.5 \mathrm{wt} \%\left(n=3\right.$, Sil- $\left.\mathrm{AN}_{3}\right)$ and $13.8 \mathrm{wt} \%$ $\left(n=21\right.$, Sil- $\left.\mathrm{AN}_{21}\right)$ of $\mathrm{AN}_{n}$ introduced onto the silica.

\section{Selectivity for Structural Isomers of Polycyclic Aro- matic Hydrocarbons}

The interaction between $\mathrm{AN}_{n}$ and polyaromatic hydrocarbons was evaluated by using retention factors in column liquid chromatography. Typical chromatograms are shown in Figure 1. The resultant retention factor $(k)$ and separation factor $(\alpha)$ were summarized in Table I, II, and III. As shown in Table I, when

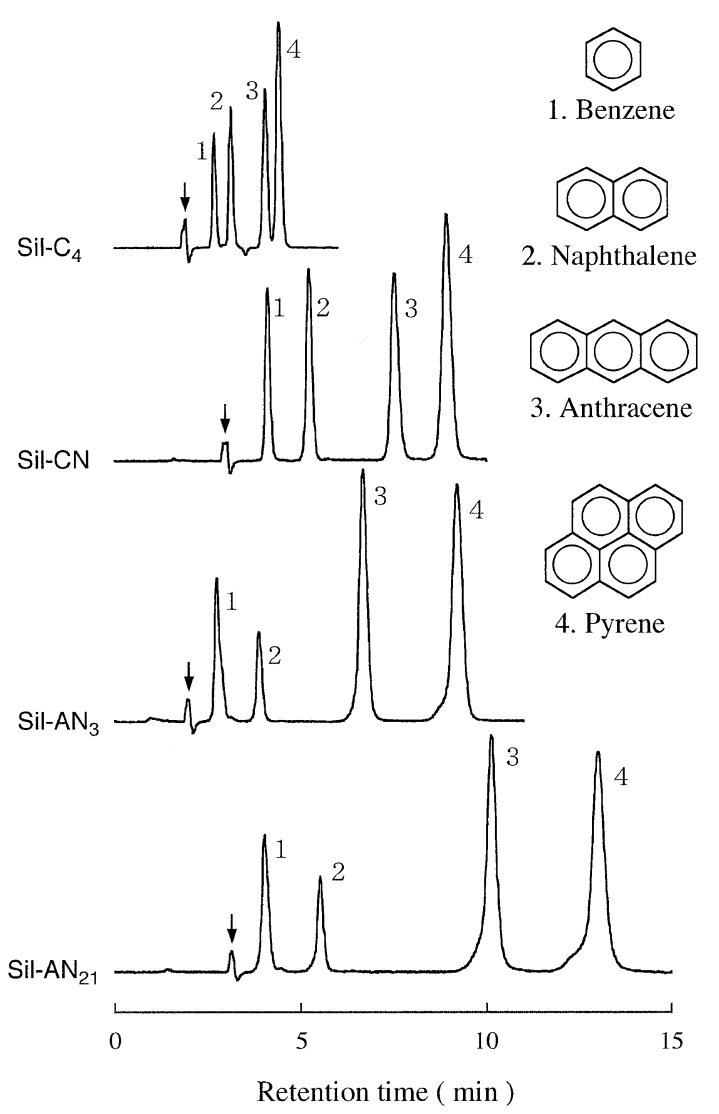

Figure 1. Typical chromatograms with $\mathrm{Sil}_{-} \mathrm{C}_{4}, \mathrm{Sil}-\mathrm{CN}$, Sil$\mathrm{AN}_{3}$, and Sil- $\mathrm{AN}_{21}$ columns. Mobile phase: methanol-water $(6: 4)$ for Sil-CN, Sil-AN ${ }_{3}$, and Sil- $\mathrm{AN}_{21}$ and methanol-water $(7: 3)$ for Sil- $\mathrm{C}_{4}$, Temperature: $35^{\circ} \mathrm{C}$, UV detection wavelength: $250 \mathrm{~nm}$, The small arrows indicate $t_{0}$. 
Table I. Retention factors $(k)$ and separation factors $(\alpha)$ for polycyclic aromatic hydrocarbons with Sil-C $4, \mathrm{Sil}_{-} \mathrm{CN}, \mathrm{Sil}_{-} \mathrm{AN}{ }_{3}$, and Sil-AN $\mathrm{AN}_{21}$. Mobile phase: methanol/water $=6 / 4\left(\mathrm{Sil}-\mathrm{CN}, \mathrm{Sil}_{-} \mathrm{AN}_{3}\right.$, and $\left.\mathrm{Sil}_{-} \mathrm{AN}_{21}\right), 7 / 3\left(\mathrm{Sil}_{-} \mathrm{C}_{4}\right)$, Flow rate: $1.0 \mathrm{~mL} \mathrm{~min}^{-1}$, Temperature: $35^{\circ} \mathrm{C}$,

UV detection wavelength: $250 \mathrm{~nm}$

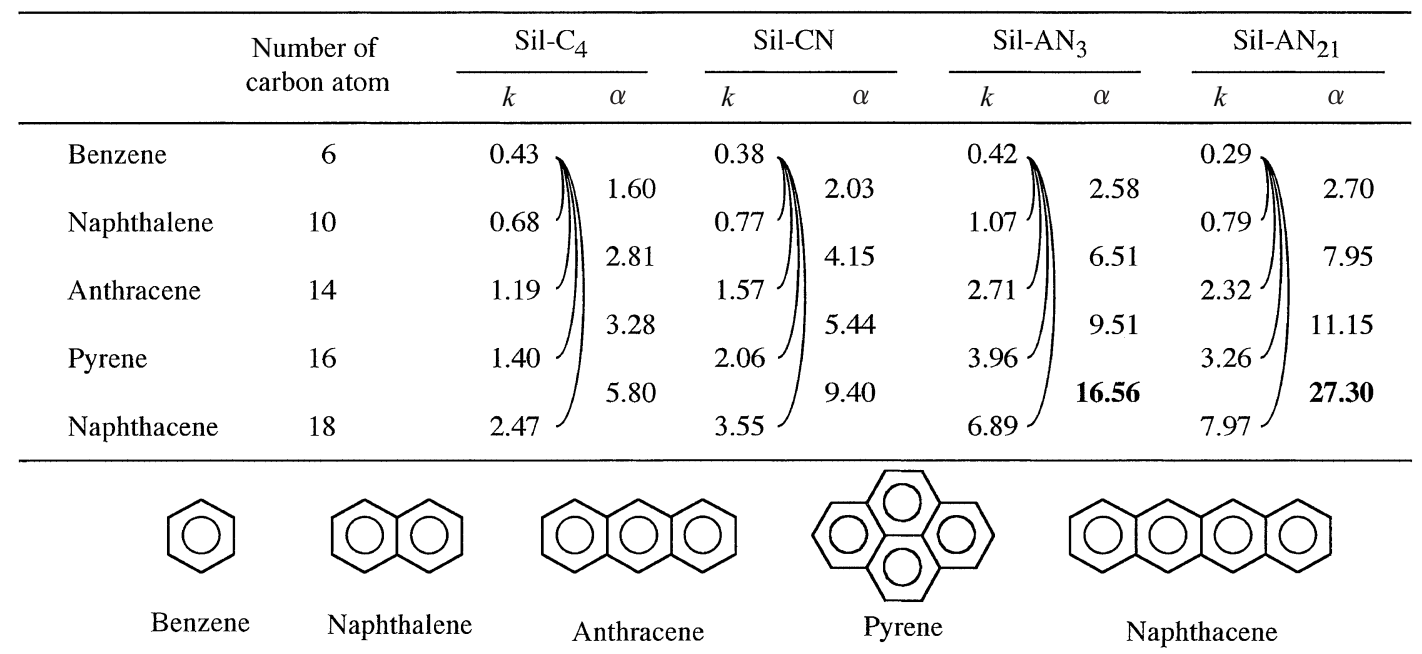

Table II. Retention factors $(k)$ and separation factors $(\alpha)$ for polycyclic aromatic hydrocarbons with Sil- $\mathrm{C}_{4}, \mathrm{Sil}_{-} \mathrm{CN}_{\text {, }} \mathrm{Sil}-\mathrm{AN}_{3}$, and Sil-AN $\mathrm{AN}_{21}$. Mobile phase: methanol/water $=6 / 4\left(\mathrm{Sil}-\mathrm{CN}, \mathrm{Sil}_{-} \mathrm{AN}_{3}\right.$, and $\left.\mathrm{Sil}-\mathrm{AN}_{21}\right), 7 / 3\left(\mathrm{Sil}_{-} \mathrm{C}_{4}\right)$, Flow rate: $1.0 \mathrm{~mL} \mathrm{~min}^{-1}$, Temperature: $35^{\circ} \mathrm{C}$, UV detection wavelength: $270 \mathrm{~nm}$

\begin{tabular}{|c|c|c|c|c|c|c|c|c|c|}
\hline & \multirow{2}{*}{$\begin{array}{l}\text { Number of } \\
\text { carbon atom }\end{array}$} & \multicolumn{2}{|c|}{ Sil-C $_{4}$} & \multicolumn{2}{|c|}{ Sil-CN } & \multicolumn{2}{|c|}{ Sil- $\mathrm{AN}_{3}$} & \multicolumn{2}{|c|}{ Sil-AN 21} \\
\hline & & $k$ & $\alpha$ & $k$ & $\alpha$ & $k$ & $\alpha$ & $k$ & $\alpha$ \\
\hline Triphenylene & 18 & 1.77 & & 2.93 & & 6.43 & & 5.16 & \\
\hline Chrysene & 18 & 2.04 & 1.15 & 3.04 & 1.04 & 6.53 ' & 1.02 & $6.48 \|$ & 1.26 \\
\hline Benz[a]anthracene & 18 & $2.19^{\prime}$ & $\begin{array}{l}1.24 \\
1.41\end{array}$ & 3.41 & $\begin{array}{l}1.16 \\
1.22\end{array}$ & 6.65 人 & $\begin{array}{l}1.05 \\
1.08\end{array}$ & 6.71 & 1.54 \\
\hline Naphthacene & 18 & 2.49 J & & 3.57 ) & & 6.92 & & 7.96 & \\
\hline
\end{tabular}

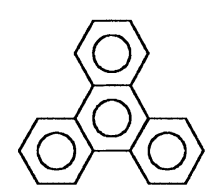

Triphenylene

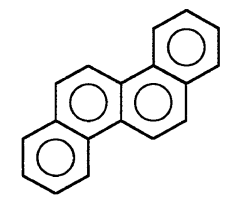

Chrysene

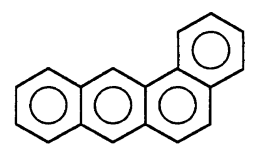

Benz[a]anthracene

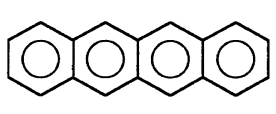

Naphthacene

Table III. Retention factors $(k)$ and separation factors $(\alpha)$ for polycyclic aromatic hydrocarbons with Sil-CN, $\mathrm{Sil}_{-} \mathrm{AN}_{3}$, and $\mathrm{Sil}-\mathrm{AN}_{21}$. Mobile phase: methanol $/$ water $=6 / 4$, Flow rate: $1.0 \mathrm{~mL} \mathrm{~min}^{-1}$, Temperature: $35^{\circ} \mathrm{C}$, UV detection wavelength: $250 \mathrm{~nm}$

\begin{tabular}{|c|c|c|c|c|c|c|}
\hline & \multicolumn{2}{|c|}{ Sil-CN } & \multicolumn{2}{|c|}{$\mathrm{Sil}^{-\mathrm{AN}_{3}}$} & \multicolumn{2}{|c|}{ Sil-AN 21} \\
\hline & $k$ & $\alpha$ & $k$ & $\alpha$ & $k$ & $\alpha$ \\
\hline Triphenylene & 2.92 & & 6.50 & & 5.22 & \\
\hline & & 1.85 & & 4.18 & & 6.56 \\
\hline$o$-Terphenyl & 1.58 & 1.61 & 1.55 & 2.19 & 0.80 & 3.30 \\
\hline$m$-Terphenyl & 2.54 & & 3.41 & & 2.62 J & \\
\hline$p$-Terphenyl & 2.79 & 1.76 & 3.50 & 2.25 & 3.21 & 4.04 \\
\hline
\end{tabular}

the samples were benzene, naphthalene, anthracene, pyrene and naphthacene, $k$ increased with an increase in the number of aromatic rings and no difference was confirmed in the retention order. However, it is clear the selectivities were much higher in Sil- $\mathrm{AN}_{n}$ than those in Sil-CN and Sil-C ${ }_{4}$. For example, $\alpha$ s between naph- thacene and benzene were 16.6 and 27.3 in Sil- $\mathrm{AN}_{3}$ and Sil- $\mathrm{AN}_{21}$ but 9.4 and 5.8 in Sil-CN and Sil-C $\mathrm{C}_{4}$. Here, it should be emphasized that $\mathrm{Sil}_{-} \mathrm{C}_{4}$ shows the smallest value. This is attributed that only Sil- $\mathrm{C}_{4}$ has no $\pi$-electron sources for $\pi-\pi$ interaction with the samples. As supported this, our previous work showed 
that Sil- $\mathrm{AN}_{n}$ was not sensitive to hydrophobicity (the number of carbon atoms) of the samples when alkyl benzenes were chosen as samples, but $\mathrm{Sil}_{-} \mathrm{C}_{4}$ showed distinct selectivity recognizing the number of carbon atoms. These results indicate that $\mathrm{AN}_{n}$ can interact with the samples through $\pi-\pi$ interaction rather than through molecular hydrophobicity. Table II emphasizes the specificity of Sil-AN ${ }_{n}$. The larger selectivity in Sil$\mathrm{AN}_{n}$ was obtained even for the aromatic compounds with a composition of $\mathrm{C}_{18} \mathrm{H}_{12}$. Water-1-octanol partition coefficient $(\log P)$ value is often used as a hydrophobic parameter. The elution order of these aromatic compounds is identical with $\log P$ value (5.25 (Triphenylene), 5.38 (Benz[a]anthracene), 5.36 (Chrysene) and 5.68 (Naphthacene)). This indicates that Sil$\mathrm{C}_{4}$ showed $\alpha \mathrm{s}$ (1.24 (Benz[a]anthracene), 1.15 (Chrysene), and 1.41 (Naphthacene) for Triphenylene) were responsible for the hydrophobicity in each compounds. On the other hand, Sil-CN and Sil-AN ${ }_{3}$ showed the lower selectivity for the structural isomers because the all isomers had the same number of $\pi$-electrons. However, it was not explained that Sil- $\mathrm{AN}_{21}$ showed the higher selectivity for these structural isomers. To answer this question, we are here proposing a multiple $\pi-$ $\pi$ interaction mechanism through polymer effect. This is derived from the facts that Sil-AN ${ }_{21}$ with higher polymerization degree always shows better selectivity for polyaromatic hydrocarbons than $\mathrm{Sil}_{-} \mathrm{AN}_{3}$ with lower polymerization degree. This assumption is furthermore discussed in the following section.

\section{Selectivity for Geometrical Isomers}

Table III shows the selectivity for several geometrical isomers. If $\mathrm{AN}_{n}$ recognizes only molecular hydrophobicity, small selectivity would be obtained for these isomers because of their small differences in molecular hydrophobicity $(\log P: 4.47$ (o-Terphenyl), 5.04 ( $m$-Terphenyl), and 5.15 ( $p$-Terphenyl)). As supported this, Sil- $\mathrm{C}_{4}$ showed very small selectivities for all the isomers listed in Table III: for example, $\alpha$ s were 1.16 ( $m$-Terphenyl $/ o$-Terphenyl), and 1.31 ( $p$-Terphenyl $/ o$ Terphenyl) in Sil-C4. Therefore, it should be noted that Sil- $\mathrm{AN}_{n}$ showed much better geometrical selectivities. As above-mentioned, $\mathrm{AN}_{n}$ interacts with $\pi$ electron-containing substances and then is not sensitive for molecular hydrophobicity, but these facts cannot explain the good geometrical selectivities because the isomers have no difference in the number of $\pi$-electrons. It seems that $\mathrm{AN}_{n}$ rather recognizes molecular shape. For example, the highest retention factor was observed in triphenylene which was almost a planar molecule. On the contrary, $p$-, $m$-, and $o$-terphenyls are not planar but rather twisted. Twisting angles of side-benzene
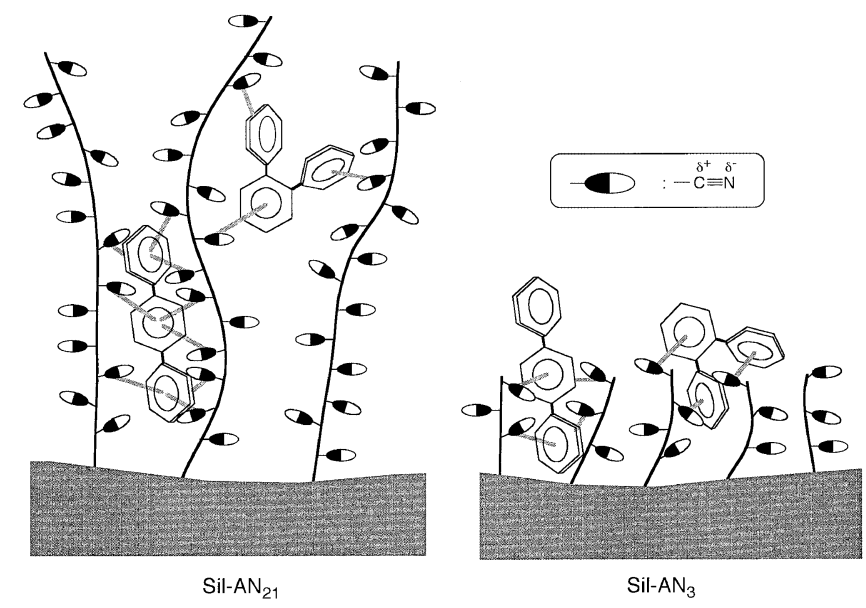

Figure 2. Schematic illustration of a multiple $\pi-\pi$ interaction mechanism of Sil- $\mathrm{AN}_{n}$. The figures indicate that a longer poly(acrylonitrile) chain provides more effective interaction area with a polycyclic aromatic hydrocarbons and recognize molecular planarity and linearity by multiple $\mathrm{CN}-\pi$-to-benzene- $\pi$ interaction through the polymer effect.

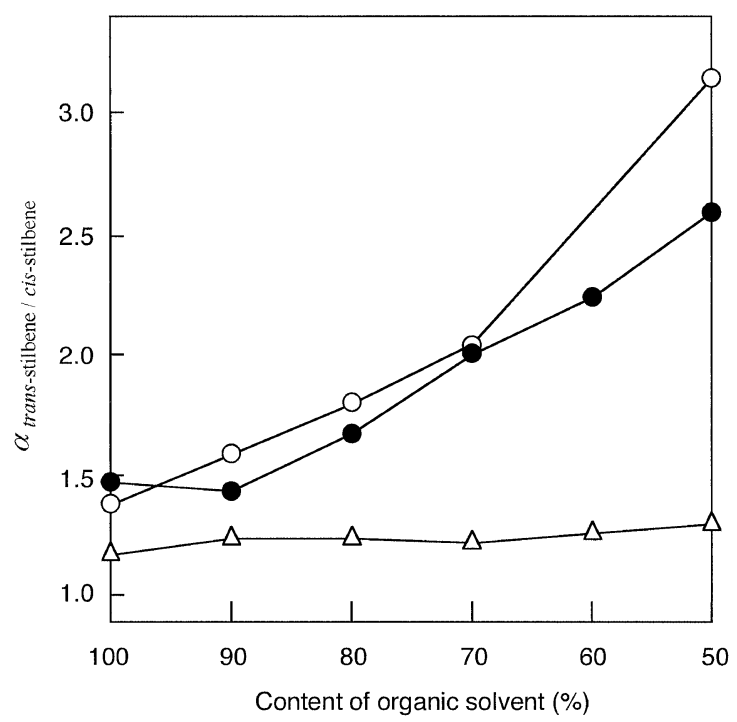

Figure 3. Effects of addition of methanol ( $\bigcirc)$, ethanol (O), and acetonitrile $(\triangle)$ to a water mixture as a mobile phase on the selectivity of stilbene isomers in Sil- $\mathrm{AN}_{21}$.

rings from molecular plane of central-benzene rings ${ }^{12}$ were calculated to be $56^{\circ}, 50^{\circ}$, and $37^{\circ}$, respectively. Interestingly, the retention factors of terphenyl isomers are smaller in relatively twisted-isomers. Stilbene has two isomers whose are trans- and cis-forms. Therefore, these isomers have the same number of $\pi$-electrons but big difference in molecular planarity. Sil- $\mathrm{C}_{4}$ provides small selectivity $(\alpha=1.02)$ but it is much better in Sil$\operatorname{AN}_{n}(\alpha=3.37$ in Sil-AN 21$)$.

On the basis of these results, we conclude that Sil$\mathrm{AN}_{n}$ recognizes not only the number of $\pi$-electrons but also the molecular planarity, but not sensitive to molecular hydrophobicity. And also we have to emphasize that these properties enhance with polymer effect 
Table IV. Retention factors $(k)$ and separation factors $(\alpha)$ for dinitrobenzene and dinitrotoluene isomers. Mobile phase: methanol/water $=5 / 5\left(\mathrm{Sil}_{-} \mathrm{C}_{4}, \mathrm{Sil}_{-\mathrm{AN}_{3}}\right.$, and Sil-AN $\left.{ }_{21}\right)$, Flow rate: $1.0 \mathrm{~mL} \mathrm{~min}^{-1}$, Temperature: $35^{\circ} \mathrm{C}$, UV detection wavelength: $250 \mathrm{~nm}$

\begin{tabular}{|c|c|c|c|c|c|c|c|c|}
\hline & \multicolumn{2}{|c|}{ Sil-C 4} & \multicolumn{2}{|c|}{ Sil-CN } & \multicolumn{2}{|c|}{ Sil-AN ${ }_{3}$} & \multicolumn{2}{|c|}{ Sil-AN 21} \\
\hline & $k$ & $\alpha$ & $k$ & $\alpha$ & $k$ & $\alpha$ & $k$ & $\alpha$ \\
\hline$p$-Dinitrobenzene & 1.011 & & 0.77 & & 0.99 & & 0.94 & \\
\hline$m$-Dinitrobenzene & $1.107 \leadsto$ & & 0.91 & 1.18 & 1.13 & 1.14 & 1.111 & 1.18 \\
\hline$o$-Dinitrobenzene & 1.097 & 1.09 & 1.302 & 1.67 & 1.13 & 1.32 & 1.232 & 1.31 \\
\hline 2,6-Dinitrotoluene & 1.147 & & 1.14 & & 1.38 & & 1.189 & \\
\hline 2,4-Dinitrotoluene & $1.237)$ & 1.08 & $1.23^{\circ}$ & 1.08 & 1.54 & 1.11 & 1.400 & 1.18 \\
\hline
\end{tabular}

because Sil-AN $\mathrm{AN}_{21}$ always shows better selectivity than Sil-AN $A_{3}$. To explain this, we are herewith proposing multiple interaction mechanisms between $\mathrm{CN}-\pi$ and benzene- $\pi$ : Figure 2 shows schematic illustrations for Sil- $\mathrm{AN}_{3}$ and Sil- $\mathrm{AN}_{21}$. A C $\equiv \mathrm{N}$ group is polarized to be $\delta^{+}$and $\delta^{-}$, respectively. This will enable hydrogen bonding of the $\delta^{-}-\mathrm{N}$ atoms with $\mathrm{OH}$ groups on silanol groups. On the other hand, the $\delta^{+}-\mathrm{C}$ atoms can interact with electron-rich aromatic rings. The interaction between $\mathrm{CN}-\pi$ and benzene- $\pi$ electrons is not yet directly detected but we can estimate it by using an inhibitor. On the separation behavior of Sil- $\mathrm{AN}_{21}$, addition of acetonitrile to a water as a mobile phase showed no change in the selectivity for stilbene isomers while addition of methanol or ethanol showed significant change in the selectivity (shown in Figure 3). This indicates that a $\mathrm{CN}$ group in acetonitrile works as an interaction source to cause the reduction of the retention time. On the basis of this assumption, $\mathrm{AN}_{21}$ is enough large to provide $\mathrm{CN}$-rich microenvironment suitable for multiple interaction with polycyclic aromatic hydrocarbons but $\mathrm{AN}_{3}$ is probably too short. In addition, it should be noted that poly(acrylonitrle) is rather rigid than flexible to be coiled. ${ }^{13}$ This explains why $\mathrm{AN}_{n}$ provides better selectivities for structural and geometrical isomers. Multiple interaction must be an advantage in recognition of isomers, especially it is effective in case of planer-to-planer and rigid-to-rigid. We have previously described this in the interaction between $\alpha$ helical poly(L-alanine) and polycyclic aromatic hydrocarbons. ${ }^{10}$ The planarity is better in $p$-terphenyl and trans-stilbene than $o$-terphenyl and cis-stilbene, respectively. Also, the multiple interaction is probably more effectively with linear compounds such as naphthacene than in bending compounds such as crysene because the interaction ability would be dominated by the contact area between host-guest molecules.

\section{Selectivity for Dinitrobenzene and Dinitrotoluene}

It is important that nitro group-containing aromatic compounds to analyze with HPLC because some of them are carcinogenic or estrogenic compounds. ${ }^{14}$ As shown in Table IV, the selectivity for dinitrobenzene isomers on Sil- $\mathrm{AN}_{n}$ were less than that on Sil-CN. It seems to be reasonable because dinitrobenzenes are so small that the molecular-shape selectivity derived from the polymer effect of poly(acrylonitrile) are not demonstrated. However, the better selectivity with Sil- $\mathrm{AN}_{n}$ was observed for dinitrotoluene isomers. It can be explained by the fact that a methyl group of dinitrotoluene works as an electron donating groups at which $\pi$-electron density in the benzene ring increases and thus Sil- $\mathrm{AN}_{n}$ can recognize the small difference of them. It is expected that Sil-AN ${ }_{n}$ can be used to separate for isomers of endocrine disturbing chemicals or agents of pharmacies because most of them are aromatic with many isomers containing donating groups.

\section{CONCLUSIONS}

The effects of polymerization degree of poly (acrylonitrile) on selective interaction for $\pi$-electron containing compounds were estimated by grafting the polymer onto silica and evaluating the retention behavior in HPLC. Sil-AN ${ }_{n}$ showed much better selectivity for polycyclic aromatic compounds with structural and geometrical isomers than simply-cyanopropylated silica (Sil-CN) and simply-hydrophobized silica (Sil-C 4 ). We have also confirmed that the selectivity increases with an increase in polymerization degree. This can be explained by multiple $\mathrm{CN}-\pi$-to-benzene- $\pi$ interaction showing the polymer effect. In closing, we wish to emphasize Sil-AN ${ }_{n}$ useful for substantial HPLC because Sil-CN with less selectivity than Sil- $\mathrm{AN}_{n}$ is still important as an unique packing materials for HPLC although compared with $\mathrm{Sil}_{-} \mathrm{C}_{4}$ and octadecylated silica have been most widely used. It will be possible that specific separations for $\pi$-electron containing compounds such as dioxins, endocrine disturbing chemicals and drugs are achieved by Sil- $\mathrm{AN}_{n}$. 


\section{REFERENCES}

1. C. Hirayama, H. Ihara, and T. Mukai, Macromolecules, 25, 6375 (1992).

2. H. Ihara, T. Fukumoto, and C. Hirayama, Anal. Sci., 9, 711 (1993).

3. T. Fukumoto, H. Ihara, S. Sakaki, H. Shosenji, and C. Hirayama, J. Chromatogr., A, 672, 237 (1994).

4. H. Ihara, H. Tanaka, S. Nagaoka, K. Sakaki, and C. Hirayama, J. Liq. Chromatogr., 19, 2967 (1996).

5. H. Ihara, S. Okazaki, K. Ohmori, S. Uemura, C. Hirayama, and S. Nagaoka, Anal. Sci., 14, 349 (1998).

6. H. Ihara, H. Tanaka, M. Shibata, S. Sasaki, and C. Hirayama, Chem. Lett., 113 (1999).

7. H. Ihara, S. Uemura, S. Okazaki, and C. Hirayama, Polym. J., 30, 394 (1998).

8. C. Hirayama, H. Ihara, S. Nagaoka, and T. Wada, Polym. J., 26, 499 (1994).

9. H. Ihara, N. Nakamura, S. Nagaoka, and C. Hirayama, Anal.
Sci., 11, 739 (1995).

10. H. Ihara, T. Nakanishi, T. Sagawa, C. Hirayama, T. Sakurai, T. Kinoshita, and Y. Tsujita, Chem. Lett., 963 (1998).

11. R. F. Rekker and H. M. de Kort, Eur. J. Med. Chem., 479, 14 (1979).

12. The chemical structures of the terphenyl isomers were estimated by Sony-Tektronix CAChe-mechanics with the MM2 option (until the energy changes were below $0.001 \mathrm{kcal}$ $\mathrm{mol}^{-1}$ ) and following CAChe-MOPAC Ver 6.00 with the PM3 option;

J. J. P. Stewart, J. Comput. Chem., 10, 221 (1989).

13. M. K. Jain and A. S. Abhiraman, J. Mater. Sci., 22, 278 (1987).

M. K. Jain, M. Balasubramanian, P. Desai, and A. S. Abhiraman, J. Mater. Sci., 22, 301 (1987).

14. H. Rosenkranz and R. Mermelstein, Mutation Res., 114, 217 (1983).

L. H. Keith, "Environmental Endocrine Disruptors: A Handbook of Property Data”, John Wiley \& Sons, Inc., New York, N.Y., 1997. 